\title{
A NATURAL APPROACH TO THE ASYMPTOTIC MEAN VALUE PROPERTY FOR THE $p$-LAPLACIAN
}

\author{
MICHINORI ISHIWATA, ROLANDO MAGNANINI, AND H. WADADE
}

Abstract. Let $1 \leq p \leq \infty$. We show that a function $u \in C\left(\mathbb{R}^{N}\right)$ is a viscosity solution to the normalized $p$-Laplace equation $\Delta_{p}^{n} u(x)=0$ if and only if the asymptotic formula

$$
u(x)=\mu_{p}(\varepsilon, u)(x)+o\left(\varepsilon^{2}\right)
$$

holds as $\varepsilon \rightarrow 0$ in the viscosity sense. Here, $\mu_{p}(\varepsilon, u)(x)$ is the $p$-mean value of $u$ on $B_{\varepsilon}(x)$ characterized as a unique minimizer of

$$
\inf _{\lambda \in \mathbb{R}}\|u-\lambda\|_{L^{p}\left(B_{\varepsilon}(x)\right)}
$$

This kind of asymptotic mean value property (AMVP) extends to the case $p=1$ previous (AMVP)'s obtained when $\mu_{p}(\varepsilon, u)(x)$ is replaced by other kinds of mean values. The natural definition of $\mu_{p}(\varepsilon, u)(x)$ makes sure that this is a monotonic and continuous (in the appropriate topology) functional of $u$. These two properties help to establish a fairly general proof of (AMVP), that can also be extended to the (normalized) parabolic $p$-Laplace equation.

\section{INTRODUCTION AND MAIN THEOREMS}

It is well-known that the classical mean value property characterizes harmonic functions and helps to derive most of their salient properties, such as weak and strong maximum principles, analyticity, Liouville's theorem, Harnack's inequality and more. In fact, we know that a continuous function $u$ is harmonic in an open set $\Omega \subseteq \mathbb{R}^{N}$ if and only if

$$
u(x)=f_{B_{\varepsilon}(x)} u(y) d y=f_{\partial B_{\varepsilon}(x)} u(y) d S_{y}
$$

for every ball $B_{\varepsilon}(x)$ with $\overline{B_{\varepsilon}(x)} \subset \Omega$; here, $f_{E} u$ denotes the mean value of $u$ over a set $E$ with respect to the relevant measure (see Evans [4] for instance). The relation (1.1) can also be regarded as a statistical characterization of solutions of the Laplace equation, without an explicit appearance of derivatives of $u$. A similar mean value property can also be obtained for linear elliptic equations with constant coefficients, by replacing balls by appropriate ellipsoids (see [2] and [3]).

Recently, starting with the work [14] of Manfredi, Parviainen and Rossi, a great attention has been paid to the so-called asymptotic mean value property (AMVP) and its applications to game theory. In [14, based on the formula

$$
f_{B_{\varepsilon}(x)} v(y) d y=v(x)+\frac{1}{2} \frac{\Delta v(x)}{N+2} \varepsilon^{2}+o\left(\varepsilon^{2}\right) \quad \text { as } \varepsilon \rightarrow 0,
$$

that holds for any smooth function $v$ not necessarily harmonic, it is shown that the characterization (1.1) for the harmonicity of $u$ can be replaced by the weaker

2010 Mathematics Subject Classification. Primary 35J60, 35K55, Secondary 35J92, 35K92.

Key words and phrases. $p$-harmonic function, asymptotic mean value property, $p$-mean value, viscosity solution for $p$-Laplace equation. 
(AMVP):

$$
u(x)=f_{B_{\varepsilon}(x)} u(y) d y+o\left(\varepsilon^{2}\right) \quad \text { as } \varepsilon \rightarrow 0,
$$

for all $x \in \Omega$.

Nonetheless, the decisive contribution of $[14$ is the observation that, provided the mean value in (1.3) is replaced by a suitable (nonlinear) statistical value related to $u$, an (AMVP) also characterizes p-harmonic functions, that is the (viscosity) solutions of the normalized $p$-Laplace equation $\Delta_{p}^{n} u=0$. Here,

$$
\Delta_{p}^{n} u=\frac{\nabla \cdot\left(|\nabla u|^{p-2} \nabla u\right)}{|\nabla u|^{p-2}} \text { for } 1 \leq p<\infty, \Delta_{\infty}^{n} u=\frac{\left\langle\nabla^{2} v \nabla v, \nabla v\right\rangle}{|\nabla v|^{2}},
$$

denotes the so-called normalized or homogeneous p-Laplacian.

In fact, in the same spirit of (1.2), for $1<p \leq \infty$ and for any smooth function with $\nabla v(x) \neq 0$, they proved the formula:

$$
\mu_{p}^{*}(\varepsilon, v)=v(x)+\frac{1}{2} \frac{\Delta_{p}^{n} v(x)}{N+p} \varepsilon^{2}+o\left(\varepsilon^{2}\right) \text { as } \varepsilon \rightarrow 0,
$$

where

$$
\mu_{p}^{*}(\varepsilon, u)=\frac{N+2}{N+p} f_{B_{\varepsilon}(x)} u(y) d y+\frac{1}{2} \frac{p-2}{N+p}\left(\frac{\max }{B_{\varepsilon}(x)} u+\frac{\min }{B_{\varepsilon}(x)} u\right) .
$$

(The average of the minimum and the maximum will be referred to as the min-max mean of $u$.)

That formula allowed them to prove that $u$ is $p$-harmonic in the viscosity sense in $\Omega$ if and only if

$$
u(x)=\mu_{p}^{*}(\varepsilon, u)+o\left(\varepsilon^{2}\right) \quad \text { as } \varepsilon \rightarrow 0,
$$

in the viscosity sense for every $x \in \Omega$ (see Section 3 for the relevant definitions), thus obtaining an (AMVP) for $p$-harmonic functions. It is also worth a mention that, for $N=2$ and small values of the parameter $p>1$, in [11 it is proved that the (AMVP) holds directly for weak solutions of the $p$-Laplace equation, without the need to interpret the formula in the viscosity sense.

Thus, the mean $\mu_{p}^{*}(\varepsilon, u)$ is an example of the desired (nonlinear) statistical value mentioned above. By similar arguments, one can obtain an (AMVP) with the ball $B_{\varepsilon}(x)$ replaced by the sphere $\partial B_{\varepsilon}(x)$ simply by replacing $(N+2) /(N+p)$ and $(p-2) /(N+p)$ by the numbers $N /(N+p-2)$ and $(p-2) /(N+p-2)$.

In the quest of extending this type of result to the case $p=1$, which is not covered by the choice (1.4), other kinds of means were proposed by several authors. Here, we mention the ones considered by Hartenstine and Rudd in [7, based on the median of a function,

$$
\begin{gathered}
\mu_{p}^{\prime}(\varepsilon, u)=\frac{1}{p} \operatorname{med}_{\partial B_{\varepsilon}(x)} u+\frac{p-1}{2 p}\left(\frac{\min }{B_{\varepsilon}(x)} u+\frac{\max }{B_{\varepsilon}(x)} u\right), \\
\mu_{p}^{\prime \prime}(\varepsilon, u)=\frac{2-p}{p} \operatorname{med}_{\partial B_{\varepsilon}(x)} u+\frac{2(p-1)}{p} f_{\partial B_{\varepsilon}(x)} u(y) d S_{y},
\end{gathered}
$$

and that considered by Kawohl, Manfredi and Parviainen in 9 ,

$$
\mu_{p}^{* *}(\varepsilon, u)=\frac{N+1}{N+p} \operatorname{av}_{\varepsilon}(u)(x)+\frac{1}{2} \frac{p-1}{N+p}\left(\frac{\min }{B_{\varepsilon}(x)} u+\frac{\max }{B_{\varepsilon}(x)} u\right),
$$


where

$$
\begin{gathered}
\operatorname{av}_{\varepsilon}(u)(x)=f_{L_{\varepsilon}} u(x+y) d S_{y}, \\
L_{\varepsilon}=\left\{y \in B_{\varepsilon}(x):(y-x) \cdot \nu=0\right\} \text { and } \\
\nu=\nu_{x, \varepsilon} \in \partial B_{1}(0) \text { is such that } u(x+\varepsilon \nu)=\frac{\min }{B_{\varepsilon}(x)} u .
\end{gathered}
$$

Both $\mu_{p}^{\prime}(\varepsilon, u)$ and $\mu_{p}^{\prime \prime}(\varepsilon, u)$ yield an (AMVP) for all the cases $1 \leq p \leq \infty$, but only when $N=2$, and $\mu_{p}^{* *}(\varepsilon, u)$ produces an (AMVP) for any $1 \leq p \leq \infty$ and $N \geq 2$.

In this paper, for $1 \leq p \leq \infty$, we propose one more mean that helps us to characterize - in an intrinsic way - $p$-harmonic functions by an (AMVP). Its definition was inspired by the simple remark that the median, the mean value and the min-max mean of a continuous function $u$ on a compact topological space $X$ equipped with a positive Radon measure $\nu$ are respectively the unique real values $\mu_{p}^{X}(u)$ that solve the variational problem

$$
\left\|u-\mu_{p}^{X}(u)\right\|_{L^{p}(X, \nu)}=\min _{\lambda \in \mathbb{R}}\|u-\lambda\|_{L^{p}(X, \nu)},
$$

for $p=1,2$, and $\infty$. Thus, it is natural to ask whether the solution of (1.9) yields a characterization of viscosity solutions of $\Delta_{p}^{n} u=0$ by means of an (AMVP), for each fixed $1 \leq p \leq \infty$.

Therefore, for each $1 \leq p \leq \infty$, we consider the $p$-mean of $u$ in $B_{\varepsilon}(x)$, that is the number defined as

$$
\mu_{p}(\varepsilon, u)(x)=\text { the unique } \mu \in \mathbb{R} \text { satisfying (1.9) with } X=\overline{B_{\varepsilon}(x)}
$$

The main result of this paper is the following characterization.

Theorem 1.1. Let $1 \leq p \leq \infty$ and let $\Omega$ be an open subset of $\mathbb{R}^{N}$. For a function $u \in C(\Omega)$ the following assertions are equivalent:

(i) $u$ is a viscosity solution of $\Delta_{p}^{n} u=0$ in $\Omega$;

(ii) $u(x)=\mu_{p}(\varepsilon, u)(x)+o\left(\varepsilon^{2}\right)$ as $\varepsilon \rightarrow 0$, in the viscosity sense for every $x \in \Omega$.

As a by-product, this theorem confirms the (AMVP) for $\mu_{p}^{\prime}(\varepsilon, u)$ and $\mu_{p}^{*}(\varepsilon, u)$ for the case $p=1$ in any dimension $N \geq 2$.

Theorem 1.1 is based on the asymptotic formula

$$
\mu_{p}(\varepsilon, v)(x)=v(x)+\frac{1}{2} \frac{\Delta_{p}^{n} v(x)}{N+p} \varepsilon^{2}+o\left(\varepsilon^{2}\right) \text { as } \varepsilon \rightarrow 0,
$$

that holds for any smooth function $v$ such that $\nabla v(x) \neq 0$.

We mention in passing that the mean $\mu_{p}^{X}(u)$ has also be considered in [5], when $p \geq 2$ and $N=2$, when $X$ is a finite set and $\nu$ is the counting measure and has proved to be effective in the numerical approximation of the operator $\Delta_{p}^{n}$. Another type of (AMVP) has been proved in [6] for $N=2$ and $1<p<\infty$; however, the mean considered there, besides the values of the function $u$ on $B_{\varepsilon}(x)$, also depends on the value of $\nabla u$ at $x$.

Compared to the means defined in (1.4), (1.6), (1.7), and (1.8) (and that in 6]), $\mu_{p}(\varepsilon, u)$ has a drawback, since it cannot be defined explicitly, unless $p=1,2, \infty$. However, it has useful properties that those means do not always have and are the consequences of the fact that $\mu_{p}(\varepsilon, u)$ is the projection of $u$ on the linear sub-space of $L^{p}\left(B_{\varepsilon}(x)\right)$ of the constant functions. As a matter of fact, we shall show that the functional $L^{p}\left(B_{\varepsilon}(x)\right) \ni u \mapsto \mu_{p}(\varepsilon, u)(x) \in \mathbb{R}$ is continuous in the corresponding $L^{p}$-topology and monotonic, in the sense that

$$
u \leq v \text { pointwise implies that } \mu_{p}(\varepsilon, u)(x) \leq \mu_{p}(\varepsilon, v)(x) .
$$


Notice that the functionals defined by $\left.\mu_{p}^{\prime} \varepsilon, u\right)$ and $\mu_{p}^{* *}(\varepsilon, u)$ are always monotonic, but never continuous for $p \in(1, \infty) \backslash\{2\}$, while those defined by $\mu_{p}^{*}(\varepsilon, u)$ and $\mu_{p}^{\prime \prime}(\varepsilon, u)$ are not always monotonic (the former for $p>2$, the latter for $1<p<2$ ) and never continuous for $p \in(1, \infty) \backslash\{2\}$, due to the presence of the min-max mean in their definition.

We shall see that the properties of continuity and monotonicity play an essential role in the proof of Theorem 1.1, since they allow to reduce the argument to the simpler case of a quadratic polynomial (see Lemma 3.1 and Theorem 3.2).

With a few technical adjustments, it is not difficult to treat the case of the parabolic $p$-Laplace operator. It is just the matter of replacing the euclidean ball and the Lebesgue measure by a suitable measure space. The appropriate choice is the so-called heat ball,

$$
E_{\varepsilon}(x, t)=\left\{(y, s) \in \mathbb{R}^{N+1}: s<t, \Phi(x-y, t-s)>\varepsilon^{-N}\right\},
$$

where

$$
\Phi(y, s)=(4 \pi s)^{-N / 2} e^{-\frac{|y|^{2}}{4 s}} \mathcal{X}_{(0, \infty)}(s) \text { for }(y, s) \in \mathbb{R}^{N} \times(-\infty, \infty)
$$

is the fundamental solution for the heat equation, equipped with the space-time measure

$$
d \nu(y, s)=\frac{|x-y|^{2}}{(t-s)^{2}} d y d s .
$$

Thus, by arguing in a similar spirit, we shall consider the value $\pi_{p}(\varepsilon, u)(x, t)$ as the unique solution of the variational problem

$$
\left\|u-\pi_{p}(\varepsilon, u)(x, t)\right\|_{L^{p}\left(E_{\varepsilon}(x, t), \nu\right)}=\min _{\lambda \in \mathbb{R}}\|u-\lambda\|_{L^{p}\left(E_{\varepsilon}(x, t), \nu\right)},
$$

Notice that the value $\pi_{p}(\varepsilon, u)(x, t)$ in (1.11) can be easily computed for $p=2$ as the caloric mean value of $u$, for which a classical mean value property holds true for solutions of the heat equation (4] $[\mathrm{pp} .52-54])$. If we define the space-time cylinder $\Omega_{T}=\Omega \times(0, T)$, we can prove the following companion of Theorem 1.1 .

Theorem 1.2. Let $1 \leq p \leq \infty$. For a function $u \in C\left(\Omega_{T}\right)$, the following assertions are equivalent:

(i) $u_{t}=\frac{N}{N+p-2} \Delta_{p}^{n} u$ in $\Omega_{T}$ in the viscosity sense;

(ii) $u(x, t)=\pi_{p}(\varepsilon, u)(x, t)+o\left(\varepsilon^{2}\right)$ as $\varepsilon \rightarrow 0$ in the viscosity sense for every $(x, t) \in$ $\Omega_{T}$.

For further developments and applications of (AMVP)'s, we refer the reader to 1], 8], 11, [12, 15], [17, and references therein.

This paper is organized as follows. In Section 2, we derive the pertinent properties of the $p$-mean value of a continuous function $u$ : continuity and monotonicity will be the most important. Then, we shall prove Theorem 1.1 and Theorem 1.2 in Sections 3 and 4 respectively. Finally, Section 5 is devoted to the calculation of some relevant integrals.

\section{Properties of $p$-Mean VAlues}

Let $X$ be a compact topological space which is also a measure space with respect to a positive Radon measure $\nu$ such that $\nu(X)<\infty$. We recall that, if $u \in C(X)$, the median $\operatorname{med}_{X} u$ of $u$ in $X$ is defined as the unique solution $\lambda$ of the equation

$$
\nu(\{y \in X: u(y) \geq \lambda\})=\nu(\{y \in X: u(y) \leq \lambda\}) .
$$

We start by showing that the definitions (1.10) and (1.11) of $\mu_{p}(\varepsilon, u)$ and $\pi_{p}(\varepsilon, u)$ are well posed. 
Theorem 2.1. Let $1 \leq p \leq \infty$ and $u \in C(X)$. There exists a unique real value $\mu_{p}^{X}(u)$ such that

$$
\left\|u-\mu_{p}^{X}(u)\right\|_{L^{p}(X), \nu}=\min _{\lambda \in \mathbb{R}}\|u-\lambda\|_{L^{p}(X, \nu)} .
$$

In particular,

$$
\begin{gathered}
\mu_{1}^{X}(u)=\operatorname{med}_{X} u, \quad \mu_{2}^{X}(u)=f_{X} u(y) d \nu \\
\text { and } \mu_{\infty}^{X}(u)=\frac{1}{2}\left(\min _{X} u+\max _{X} u\right) .
\end{gathered}
$$

Furthermore, for $1 \leq p<\infty, \mu_{p}^{X}(u)$ is characterized by the equation

$$
\int_{X}\left|u(y)-\mu_{p}^{X}(u)\right|^{p-2}\left[u(y)-\mu_{p}^{X}(u)\right] d \nu=0,
$$

where, for $1 \leq p<2$, we mean that the integrand is zero if $u(y)-\mu_{p}^{X}(u)=0$.

Proof. The case $p=1$ is a straightforward extension of the proofs in [16], 18] and [19].

If $p=\infty$, the assertion follows at once by observing that

$$
\max _{X}|u-\lambda|=\max \left(\max _{X} u-\lambda, \lambda-\min _{X} u\right) .
$$

Next, in the case $1<p<\infty$, we observe that

$$
\min _{\lambda \in \mathbb{R}}\|u-\lambda\|_{L^{p}(X, \nu)}=\min _{v \in \Lambda}\|u-v\|_{L^{p}(X, \nu)},
$$

where $\Lambda$ is the subspace of constant functions on $X$; in other words $\mu_{p}^{X}(u)$ is a projection of $u$ on $\Lambda$. Thus, the existence, uniqueness and characterization of $\mu_{p}^{X}(u)$ are guaranteed by the theorem of the projection, since $L^{p}(X, \nu)$ is uniformly convex and $\Lambda$ is a closed subspace, and the differentiability of the function $\lambda \mapsto$ $\|u-\lambda\|_{L^{p}(X, \nu)}($ see [10]).

The expression of $\mu_{2}^{X}(u)$ is readily computed as the minimum point of a quadratic polynomial.

Remark 2.2. Note that, for $1<p \leq \infty$, Theorem 2.1 extends to the case in which $u \in L^{p}(X, \nu)$, provided the minimum and the maximum are replaced by

$$
\underset{X}{\operatorname{essinf}} u \text { and } \underset{X}{\operatorname{ess} \sup } u \text {. }
$$

If $u \in L^{1}(X, \nu) \backslash C(X)$, it is known that the median of $u$ in $X$ may not be unique (see [16]).

The following corollary will be very useful for further computations. We set $B=B_{1}(0)$.

Corollary 2.3. Let $u \in L^{p}\left(B_{\varepsilon}(x)\right)$, for $1<p \leq \infty$, and $u \in C\left(B_{\varepsilon}(x)\right)$, for $p=1$. If we let $u_{\varepsilon}(z)=u(x+\varepsilon z)$ for $z \in \bar{B}$ and set

then it holds that

$$
\mu_{p}(\varepsilon, u)(x)=\mu_{p}^{B_{\varepsilon}(x)}(u),
$$

Proof. It suffices to observe that, for every $\lambda \in \mathbb{R}$, it holds that

$$
\|u-\lambda\|_{L^{p}\left(B_{\varepsilon}(x)\right)}=\varepsilon^{N / p}\left\|u_{\varepsilon}-\lambda\right\|_{L^{p}(B)},
$$

for $1 \leq p<\infty$, and

$$
\|u-\lambda\|_{L^{\infty}\left(B_{\varepsilon}(x)\right)}=\left\|u_{\varepsilon}-\lambda\right\|_{L^{\infty}(B)},
$$


and hence invoke the uniqueness part of Theorem 2.1

In the next two theorems we regard $\mu_{p}^{X}(u)$ as the value at $u$ of a functional $\mu_{p}^{X}$ on $L^{p}(X)$. If $p=1$ and $u \in L^{1}(X) \backslash C(X)$, we allow $\mu_{1}^{X}(u)$ to be any minimizing value of $\lambda \mapsto\|u-\lambda\|_{L^{1}(X)}$ on $\mathbb{R}$, whenever it is convenient.

Theorem 2.4 (Continuity). Let $1 \leq p \leq \infty$. It holds that

$$
\left|\left\|u-\mu_{p}^{X}(u)\right\|_{L^{p}(X)}-\left\|v-\mu_{p}^{X}(v)\right\|_{L^{p}(X)}\right| \leq\|u-v\|_{L^{p}(X)},
$$

for any $u, v \in L^{p}(X)$.

Moreover, if $u_{n} \rightarrow u$ in $L^{p}(X)$ for $1 \leq p \leq \infty$ and $u_{n}, u \in C(X)$ for $p=1$, then $\mu_{p}^{X}\left(u_{n}\right) \rightarrow \mu_{p}^{X}(u)$ as $n \rightarrow \infty$.

In particular, the same conclusion holds for any $p \in[1, \infty]$, if $\left\{u_{n}\right\}_{n \in \mathbb{N}} \subset C(X)$ converges to $u$ uniformly on $X$ as $n \rightarrow \infty$.

Proof. The inequality (2.5) simply follows by observing that $\left\|u-\mu_{p}^{X}(u)\right\|_{L^{p}(X)}$ is nothing else than the distance of $u$ from the subspace $\Lambda$.

Next, if $u_{n} \rightarrow u$ in $L^{p}(X)$ as $n \rightarrow \infty$, 2.5 implies that

$$
\left\|u_{n}-\mu_{p}^{X}\left(u_{n}\right)\right\|_{L^{p}(X)} \rightarrow\left\|u-\mu_{p}^{X}(u)\right\|_{L^{p}(X)} \text { as } n \rightarrow \infty .
$$

We conclude by observing that, since $\mu_{p}^{X}(u)$ unique for $1<p \leq \infty$ and for $p=$ 1 if $u \in C(X)$, any converging sub-sequence of $\left\{\mu_{p}^{X}\left(u_{n}\right)\right\}_{n \in \mathbb{N}}$ must converge to $\mu_{p}^{X}(u)$.

Theorem 2.5 (Monotonicity). Let $u$ and $v$ be two functions in $L^{p}(X)$, for $1<$ $p \leq \infty$, or in $C(X)$ for $p=1$.

If $u \leq v$ a.e. on $L^{p}(X)$, then $\mu_{p}^{X}(u) \leq \mu_{p}^{X}(v)$.

Proof. For $1<p<\infty$, we observe that the function $F: \mathbb{R} \times \mathbb{R} \rightarrow \mathbb{R}$ defined by

$$
F(u, \lambda)=|u-\lambda|^{p-2}(u-\lambda)
$$

is increasing in $u$ for fixed $\lambda$ and decreasing in $\lambda$ for fixed $u$; hence,

$$
\int_{X}|u(y)-\lambda|^{p-2}[u(y)-\lambda] d \nu_{y} \leq \int_{X}|v(y)-\lambda|^{p-2}[v(y)-\lambda] d \nu_{y}
$$

if $u \leq v$ a.e. in $X$. The characterization (2.2) then yields that $\mu_{p}^{X}(u) \leq \mu_{p}^{X}(v)$.

Next, we know that

$$
\mu_{\infty}^{X}(u)=\frac{1}{2}\{\underset{X}{\operatorname{essinf}} u+\underset{X}{\operatorname{ess} \sup } u\}
$$

thus, the conclusion follows by an inspection.

Finally, we know that $\mu_{1}^{X}(u)$ is the unique zero of the function defined by

$$
F_{u}(\lambda)=\nu(\{y \in X: u(y) \geq \lambda\})-\nu(\{y \in X: u(y) \leq \lambda\}), \lambda \in \mathbb{R} .
$$

The conclusion then follows by observing that $F_{u} \leq F_{v}$.

Remark 2.6. Notice that the mean $\mu_{p}^{m p r}(\varepsilon, u)$ in (1.4) is not monotonic when $1 \leq p<2$ and is continuous in $L^{p}\left(B_{\varepsilon}(x)\right)$ only for $p=2, \infty$.

The mean $\mu_{p}^{h r, 2}(\varepsilon, u)$ in (1.7) is not monotonic for $2<p<\infty$ and the mean $\mu_{p}^{h r, 1}(\varepsilon, u)$ in (1.6) is not continuous unless $p=\infty$.

Finally, the mean $\mu_{p}^{k m p}(\varepsilon, u)$ in (1.8) is not continuous unless $p=\infty$.

To disprove continuity, it is sufficient to take the sequence of functions $u_{n}(y)=$ $(|y-x| / \varepsilon)^{n}$ for $y \in B_{\varepsilon}(x)$ : this converges to zero in $L^{p}\left(B_{\varepsilon}(x)\right)$ for $1 \leq p<\infty$, but the average of its maximum and minimum is always $1 / 2$.

The proof of following proposition is straightforward. 
Proposition 2.7. We have that

(i) $\mu_{p}^{X}(u+c)=c+\mu_{p}^{X}(u)$ for every $c \in \mathbb{R}$;

(ii) $\mu_{p}^{X}(\alpha u)=\alpha \mu_{p}^{X}(u)$ for every $\alpha \in \mathbb{R}$.

\section{The (AMVP) FOR THE ELLIPTIC CASE}

This section is devoted to prove Theorem [1.1. We first give a proof of the (AMVP) for smooth functions. The following lemma is the crucial step of that proof.

Lemma 3.1. Let $1 \leq p \leq \infty$, pick $\xi \in \mathbb{R}^{N} \backslash\{0\}$, and let $A$ be a symmetric $N \times N$ matrix. Consider the quadratic function $q: B_{\varepsilon}(x) \rightarrow \mathbb{R}$ defined by

$$
q(y)=q(x)+\xi \cdot(y-x)+\frac{1}{2}\langle A(y-x), y-x\rangle, y \in B_{\varepsilon}(x) .
$$

Then it holds that

$$
\mu_{p}(\varepsilon, q)(x)=q(x)+\frac{1}{2(N+p)}\left\{\operatorname{tr}(A)+(p-2) \frac{\langle A \xi, \xi\rangle}{|\xi|^{2}}\right\} \varepsilon^{2}+o\left(\varepsilon^{2}\right)
$$

as $\varepsilon \rightarrow 0$.

Proof. Set

$$
q_{\varepsilon}(z)=q(x+\varepsilon z), \quad v_{\varepsilon}(z)=\frac{q(x+\varepsilon z)-q(x)}{\varepsilon}, \quad \text { and } \quad v(z)=\xi \cdot z .
$$

We know that

thus, Proposition 2.7 implies that

$$
\mu_{p}(\varepsilon, q)(x)=\mu_{p}\left(1, q_{\varepsilon}\right)(0)
$$

$$
\frac{\mu_{p}(\varepsilon, q)(x)-q(x)}{\varepsilon}=\mu_{p}\left(1, v_{\varepsilon}\right)(0),
$$

and $v_{\varepsilon}$ converges to $v$ uniformly on $B$ as $\varepsilon \rightarrow 0$. Theorem 2.4 then yields that

$$
\lim _{\varepsilon \rightarrow 0} \frac{\mu_{p}(\varepsilon, q)(x)-q(x)}{\varepsilon}=\mu_{p}(1, v)(0),
$$

and $\lambda=\mu_{p}(1, v)(0)=0,0$ is the unique root of the equation

$$
\int_{B}|v(z)-\lambda|^{p-2}[v(z)-\lambda] d z=0
$$

for $1 \leq p<\infty$, and for $p=\infty$ maximizes the quantity

$$
\max (|\xi|-\lambda, \lambda+|\xi|)
$$

for $\lambda \in \mathbb{R}$.

For $1 \leq p \leq \infty$, set

$$
\delta_{\varepsilon}=\frac{\mu_{p}(\varepsilon, q)(x)-q(x)}{\varepsilon^{2}} .
$$

Case $\mathbf{1}<\mathbf{p}<\infty$. We define the function $h(s)=|s|^{p-2} s$; by some manipulations, we get that

$$
\int_{B} h\left(\xi \cdot z+\varepsilon\left[\langle A z, z\rangle / 2-\delta_{\varepsilon}\right]\right) d z=0
$$

Without loss of generality, we assume that $|\xi|=1$, apply the change of variables $z=R y$, where $R$ is a rotation matrix such that ${ }^{t} R \xi=e_{1}$, and set $C={ }^{t} R A R$ to obtain that

$$
\int_{B} \frac{h\left(y_{1}+\varepsilon\left[\langle C y, y\rangle / 2-\delta_{\varepsilon}\right]\right)-h\left(y_{1}\right)}{\varepsilon} d y=0,
$$


since $\int_{B} h\left(y_{1}\right) d y=0$. Thus, we have that

$$
\int_{B}\left\{\int_{0}^{1} h^{\prime}\left(y_{1}+\tau \varepsilon\left[\langle C y, y\rangle / 2-\delta_{\varepsilon}\right]\right) d \tau\right\}\left[\langle C y, y\rangle / 2-\delta_{\varepsilon}\right] d y=0,
$$

and this implies that $\delta_{\varepsilon}$ is bounded by some constant $c$ ( $c$ is equal to half of the norm of the matrix $C$ ).

If $2 \leq p<\infty$, it is easy to prove that, by the dominated convergence theorem, (any converging subsequence of) $\delta_{\varepsilon}$ converges to the number $\delta_{0}$ defined by

$$
\int_{B} h^{\prime}\left(y_{1}\right)\left[\langle C y, y\rangle / 2-\delta_{0}\right] d y=0 .
$$

If $1<p<2$, we observe that

$$
\left|\int_{0}^{1} h^{\prime}\left(y_{1}+\tau \varepsilon\left[\langle C y, y\rangle / 2-\delta_{\varepsilon}\right]\right)\left[\langle C y, y\rangle / 2-\delta_{\varepsilon}\right] d \tau\right| \leq 2 c|| y_{1}|-2 c \varepsilon|^{p-2}
$$

and

$$
\lim _{\varepsilon \rightarrow 0} \int_{B}|| y_{1}|-2 c \varepsilon|^{p-2} d y=\int_{B}\left|y_{1}\right|^{p-2} d y .
$$

If (any converging subsequence of) $\delta_{\varepsilon}$ converges to a number $\delta_{0}$, then the integrand in (3.2) converges pointwise to $h^{\prime}\left(y_{1}\right)\left[\langle C y, y\rangle / 2-\delta_{0}\right]$, and hence we can conclude that (3.3) holds, by the generalized dominated convergence theorem (Theorem 5.4).

Therefore, by Lemma 5.1 we have that

$$
\begin{aligned}
\lim _{\varepsilon \rightarrow 0} \delta_{\varepsilon}=\frac{1}{2} \frac{\int_{B}\left|y_{1}\right|^{p-2}\langle C y, y\rangle d y}{\int_{B}\left|y_{1}\right|^{p-2} d y} & = \\
\frac{1}{2(N+p)}\left\{\operatorname{tr}(C)+(p-2)\left\langle C e_{1}, e_{1}\right\rangle\right\}= & \frac{1}{2(N+p)}\left\{\operatorname{tr}(A)+(p-2) \frac{\langle A \xi, \xi\rangle}{|\xi|^{2}}\right\},
\end{aligned}
$$

since $\left\langle C e_{1}, e_{1}\right\rangle=\left\langle A R e_{1}, R e_{1}\right\rangle$, with $R e_{1}=\xi /|\xi|$.

Case $\mathbf{p}=\mathbf{1}$. We know that $\mu_{1}(\varepsilon, q)$ is the unique root of the equation

$$
\left|\left\{y \in B_{\varepsilon}(x): q(y)>\mu_{1}(\varepsilon, q)\right\}\right|=\left|\left\{y \in B_{\varepsilon}(x): q(y)<\mu_{1}(\varepsilon, q)\right\}\right| .
$$

Next, manipulating (3.4) gives that

$$
\left|\left\{z \in B: \xi \cdot z+\frac{\varepsilon}{2}\langle A z, z\rangle>\varepsilon \delta_{\varepsilon}\right\}\right|=\left|\left\{z \in B: \xi \cdot z+\frac{\varepsilon}{2}\langle A z, z\rangle<\varepsilon \delta_{\varepsilon}\right\}\right|
$$

and, by applying the substitution $z=R y$, where $R$ is a rotation matrix such that ${ }^{t} R \xi=|\xi| e_{1}$, we can infer that

$$
\begin{aligned}
\left|\left\{y \in B:|\xi| y_{1}+\frac{\varepsilon}{2}\langle C y, y\rangle>\varepsilon \delta_{\varepsilon}\right\}\right| & = \\
& \left|\left\{y \in B:|\xi| y_{1}+\frac{\varepsilon}{2}\langle C y, y\rangle<\varepsilon \delta_{\varepsilon}\right\}\right|,
\end{aligned}
$$

where $C={ }^{t} R A R$.

Now, consider the right-hand side of the last formula, set

$$
f_{\varepsilon}(y)=|\xi| y_{1}+\frac{\varepsilon}{2}\langle C y, y\rangle
$$

and

$$
c_{\varepsilon}=\left|\left\{y \in B: f_{\varepsilon}(y)<\varepsilon \delta_{\varepsilon}\right\}\right|-\left|B^{-}\right|,
$$

where $B^{-}=\left\{y \in B: y_{1} \leq 0\right\}$. The use of the change of variables

$$
y=\frac{\varepsilon z_{1}}{|\xi|} e_{1}+z^{\prime} \text { where } z^{\prime}=\left(0, z_{2}, \ldots, z_{N}\right)
$$


yields that

$$
\frac{|\xi|}{\varepsilon} c_{\varepsilon}=\left|\left\{\left(z_{1}, z^{\prime}\right) \in B^{\varepsilon}: \varepsilon^{-1} f_{\varepsilon}\left(\frac{\varepsilon z_{1}}{|\xi|} e_{1}+z^{\prime}\right)<\delta_{\varepsilon}\right\}\right|-\left|B^{\varepsilon,-}\right|,
$$

where

$$
B^{\varepsilon}=\left\{\left(z_{1}, z^{\prime}\right) \in \mathbb{R}^{N}:\left(\varepsilon z_{1} /|\xi|\right)^{2}+\left|z^{\prime}\right|^{2}<1\right\}, \quad \text { and } B^{\varepsilon,-}=\left\{z \in B^{\varepsilon}: z_{1} \leq 0\right\} .
$$

Now, set $B^{\prime}=\left\{z^{\prime} \in \mathbb{R}^{N-1}:\left|z^{\prime}\right|<1\right\}$ and notice that, if $\varepsilon$ is small enough, by the implicit function theorem, there is a unique function $g_{\varepsilon}: B^{\prime} \rightarrow \mathbb{R}$ such that

$$
\varepsilon^{-1} f_{\varepsilon}\left(\frac{\varepsilon g_{\varepsilon}\left(z^{\prime}\right)}{|\xi|} e_{1}+z^{\prime}\right)=\delta_{\varepsilon} \text { for } z^{\prime} \in B^{\prime}
$$

We can then infer that

$$
\begin{aligned}
& c_{\varepsilon}=\frac{\varepsilon}{|\xi|} \int_{B^{\prime}}\left\{\min \left[g_{\varepsilon}^{+}\left(z^{\prime}\right),(|\xi| / \varepsilon) \sqrt{1-\left|z^{\prime}\right|^{2}}\right]\right.- \\
&\left.\min \left[g_{\varepsilon}^{-}\left(z^{\prime}\right),(|\xi| / \varepsilon) \sqrt{1-\left|z^{\prime}\right|^{2}}\right]\right\} d z^{\prime},
\end{aligned}
$$

where $g_{\varepsilon}^{+}$and $g_{\varepsilon}^{-}$denote the positive and negative parts of $g_{\varepsilon}$. Thus, since $g_{\varepsilon}\left(z^{\prime}\right) \rightarrow$ $\delta_{0}-\left\langle C z^{\prime}, z^{\prime}\right\rangle / 2$ pointwise (possibly passing to a subsequence), by the dominated convergence theorem, we obtain that

$$
\lim _{\varepsilon \rightarrow 0} \frac{|\xi|}{\varepsilon} c_{\varepsilon}=\int_{B^{\prime}}\left[\delta_{0}-\frac{1}{2}\left\langle C z^{\prime}, z^{\prime}\right\rangle\right] d z^{\prime} .
$$

We can repeat the same arguments for the left-hand side of (3.5) and obtain that

$$
\lim _{\varepsilon \rightarrow 0} \frac{|\xi|}{\varepsilon}\left\{\left|\left\{y \in B: f_{\varepsilon}(y)>\varepsilon \delta_{\varepsilon}\right\}\right|-\left|B^{+}\right|\right\}=\int_{B^{\prime}}\left[\frac{1}{2}\left\langle C z^{\prime}, z^{\prime}\right\rangle-\delta_{0}\right] d z^{\prime} .
$$

Therefore, (3.5) implies that

$$
\int_{B^{\prime}}\left[\delta_{0}-\frac{1}{2}\left\langle C z^{\prime}, z^{\prime}\right\rangle\right] d z^{\prime}=0
$$

and hence

$$
\delta_{0} \frac{\omega_{N-1}}{N-1}=\frac{1}{2} \int_{B^{\prime}}\left\langle C z^{\prime}, z^{\prime}\right\rangle d z^{\prime}=\frac{\omega_{N-1}}{2\left(N^{2}-1\right)} \sum_{j=2}^{N} C_{j j} .
$$

Finally, the desired conclusion follows from

$$
\lim _{\varepsilon \rightarrow 0} \frac{\mu_{1}(\varepsilon, q)(x)-q(x)}{\varepsilon^{2}}=\lim _{\varepsilon \rightarrow 0} \delta_{\varepsilon}=\delta_{0},
$$

where

$$
2(N+1) \delta_{0}^{2}=\operatorname{tr}(C)-\left\langle C e_{1}, e_{1}\right\rangle=\operatorname{tr}(A)-\frac{\langle A \xi, \xi\rangle}{|\xi|^{2}},
$$

since $\left\langle C e_{1}, e_{1}\right\rangle=\left\langle A R e_{1}, R e_{1}\right\rangle$, with $R e_{1}=\xi /|\xi|$.

Case $\mathbf{p}=\infty$. For what we already showed at the beginning of this proof, we know that

$$
\begin{aligned}
\frac{\mu_{\infty}(\varepsilon, q)(x)-q(x)}{\varepsilon}= & \\
& \frac{1}{2}\left\{\min _{z \in B}[\xi \cdot z+\varepsilon\langle A z, z\rangle / 2]+\max _{z \in B}[\xi \cdot z+\varepsilon\langle A z, z\rangle / 2]\right\} .
\end{aligned}
$$


Now, notice that, if $\varepsilon$ is sufficiently small, the minimum and the maximum are respectively attained at the points $z_{\varepsilon}^{+}$and $z_{\varepsilon}^{-}$on $\partial B$ and

$$
z_{\varepsilon}^{ \pm}= \pm \frac{\xi+\varepsilon A z_{\varepsilon}^{\prime}}{\left|\xi+\varepsilon A z_{\varepsilon}^{\prime}\right|}= \pm \frac{\xi}{|\xi|}+o(\varepsilon)
$$

as $\varepsilon \rightarrow 0$. Thus, we can infer that

$$
\frac{\mu_{\infty}(\varepsilon, q)-q(x)}{\varepsilon^{2}}=\frac{\left\langle A z_{\varepsilon}^{+}, z_{\varepsilon}^{+}\right\rangle+\left\langle A z_{\varepsilon}^{-}, z_{\varepsilon}^{-}\right\rangle}{4}+o(1)
$$

and conclude that

$$
\frac{\mu_{\infty}(\varepsilon, q)-q(x)}{\varepsilon^{2}} \rightarrow \frac{1}{2} \frac{\langle A \xi, \xi\rangle}{|\xi|^{2}}
$$

as $\varepsilon \rightarrow 0$.

Theorem 3.2 (Asymptotics for $\mu_{p}(\varepsilon, u)$ as $\varepsilon \rightarrow 0$ ). Let $1 \leq p \leq \infty$. Let $\Omega \subseteq \mathbb{R}^{N}$ be an open set and $x \in \Omega$.

If $u \in C^{2}(\Omega)$ with $\nabla u(x) \neq 0$, then

$$
\mu_{p}(\varepsilon, u)(x)=u(x)+\frac{1}{2} \frac{\Delta_{p}^{n} u(x)}{N+p} \varepsilon^{2}+o\left(\varepsilon^{2}\right) \text { as } \varepsilon \rightarrow 0 .
$$

Proof. Let $\varepsilon>0$ be such that $\overline{B_{\varepsilon}(x)} \subset \Omega$ and consider the function $q(y)$ in Lemma 3.1 with $q(x)=u(x), \xi=\nabla u(x)$ and $A=\nabla^{2} u(x)$; also, notice that

$$
\operatorname{tr}(A)+(p-2) \frac{\langle A \xi, \xi\rangle}{|\xi|^{2}}=\Delta_{p}^{n} u(x) .
$$

Set $u_{\varepsilon}(z)=u(x+\varepsilon z)$ and $q_{\varepsilon}(z)=q(x+\varepsilon z)$; since $u \in C^{2}(\Omega)$, then for every $\eta>0$ there exists $\varepsilon_{\eta}>0$ such that

$$
\left|u_{\varepsilon}(z)-q_{\varepsilon}(z)\right|<\eta \varepsilon^{2} \text { for every } z \in \bar{B} \text { and } 0<\varepsilon<\varepsilon_{\eta} .
$$

Thus, since by Proposition 2.7

$$
\mu_{p}\left(\varepsilon, q \pm \eta \varepsilon^{2}\right)(x)=\mu_{p}(\varepsilon, q)(x) \pm \eta \varepsilon^{2},
$$

Theorem 2.5 and Corollary 2.3 yield that

$$
\frac{\mu_{p}(\varepsilon, q)(x)-u(x)}{\varepsilon^{2}}-\eta \leq \frac{\mu_{p}(\varepsilon, u)(x)-u(x)}{\varepsilon^{2}} \leq \frac{\mu_{p}(\varepsilon, q)(x)-u(x)}{\varepsilon^{2}}+\eta .
$$

Therefore, Lemma 3.1 implies that

$$
\begin{aligned}
\frac{1}{2} \frac{\Delta_{p}^{n} u(x)}{N+p}-\eta \leq \liminf _{\varepsilon \rightarrow 0} \frac{\mu_{p}(\varepsilon, u)(x)-u(x)}{\varepsilon^{2}} & \leq \\
& \limsup _{\varepsilon \rightarrow 0} \frac{\mu_{p}(\varepsilon, u)(x)-u(x)}{\varepsilon^{2}} \leq \frac{1}{2} \frac{\Delta_{p}^{n} u(x)}{N+p}+\eta .
\end{aligned}
$$

The desired conclusion follows, since $\eta$ is arbitrary.

Corollary 3.3 ((AMVP) for smooth functions). Let $1 \leq p \leq \infty$ and $u \in C^{2}(\Omega)$. The following assertions are equivalent:

(i) $\Delta_{p}^{n} u(x)=0$ at any $x \in \Omega$ such that $\nabla u(x) \neq 0$;

(ii) $u(x)=\mu_{p}(\varepsilon, u)(x)+o\left(\varepsilon^{2}\right)$ as $\varepsilon \rightarrow 0$ at any $x \in \Omega$ such that $\nabla u(x) \neq 0$.

Remark 3.4. Without any essential modification, we can show a similar result with $\mu_{p}(\varepsilon, u)(x)$ in Corollary 3.3 replaced by an analogous spherical $p$-mean value of $u$ on $\partial B_{\varepsilon}(x)$, that is the minimum value in the variational problem (1.9), where the $L^{p}$ norm is taken on $\partial B_{\varepsilon}(x)$. The asymptotic formula (3.6) reads in this case as:

$$
\mu_{p}(\varepsilon, u)(x)=u(x)+\frac{1}{2} \frac{\Delta_{p}^{n} u(x)}{N+p-2} \varepsilon^{2}+o\left(\varepsilon^{2}\right) \text { as } \varepsilon \rightarrow 0 .
$$


We are now going to prove that continuous viscosity solutions of the normalized $p$-Laplace equation are characterized by an (AMVP) in the viscosity sense. We recall the relevant definitions from [14].

A function $u \in C(\Omega)$ is a viscosity solution of $\Delta_{p}^{n} u=0$ in $\Omega$, if both of the following requisites hold at every $x \in \Omega$ :

(i) for any function $\phi$ of class $C^{2}$ near $x$ such that $u-\phi$ has a strict minimum at $x$ with $u(x)=\phi(x)$ and $\nabla \phi(x) \neq 0$, there holds that $\Delta_{p}^{n} \phi(x) \leq 0$;

(ii) for any function $\phi$ of class $C^{2}$ near $x$ such that $u-\phi$ has a strict maximum at $x$ with $u(x)=\phi(x)$ and $\nabla \phi(x) \neq 0$, there holds that $\Delta_{p}^{n} \phi(x) \geq 0$.

We say that a function $u \in C(\Omega)$ satisfies at $x \in \Omega$ the asymptotic mean value property (AMVP)

$$
u(x)=\mu_{p}(\varepsilon, u)(x)+o\left(\varepsilon^{2}\right) \text { as } \varepsilon \rightarrow 0
$$

in the viscosity sense if both of the following requisites hold:

(a) for any function $\phi$ of class $C^{2}$ near $x$ such that $u-\phi$ has a strict minimum at $x$ with $u(x)=\phi(x)$ and $\nabla \phi(x) \neq 0$, there holds that

$$
\phi(x) \geq \mu_{p}(\varepsilon, \phi)(x)+o\left(\varepsilon^{2}\right) \text { as } \varepsilon \rightarrow 0 ;
$$

(b) for any function $\phi$ of class $C^{2}$ near $x$ such that $u-\phi$ has a strict maximum at $x$ with $u(x)=\phi(x)$ and $\nabla \phi(x) \neq 0$, there holds that

$$
\phi(x) \leq \mu_{p}(\varepsilon, \phi)(x)+o\left(\varepsilon^{2}\right) \text { as } \varepsilon \rightarrow 0 .
$$

We are now in the position to prove Theorem 1.1

Proof of Theorem 1.1. Let $\phi$ be of class $C^{2}$ near $x$ with $\nabla \phi(x) \neq 0$; by Theorem 3.2 , we know that

$$
\phi(x)=\mu_{p}(\varepsilon, \phi)(x)-\frac{1}{2} \frac{\varepsilon^{2}}{N+p} \Delta_{p}^{n} \phi(x)+o\left(\varepsilon^{2}\right)
$$

as $\varepsilon \rightarrow 0$.

Thus, if $u-\phi$ has a strict minimum at $x$ with $u(x)=\phi(x)$ and $\Delta_{p}^{n} \phi(x) \leq 0$, then (3.7) implies that

$$
\phi(x) \geq \mu_{p}(\varepsilon, \phi)(x)+o\left(\varepsilon^{2}\right) \text { as } \varepsilon \rightarrow 0 .
$$

Conversely, if $\phi(x) \geq \mu_{p}(\varepsilon, \phi)(x)+o\left(\varepsilon^{2}\right)$ as $\varepsilon \rightarrow 0$, by (3.7) we infer that

$$
-\Delta_{p}^{n} \phi(x) \geq o(1) \quad \text { as } \varepsilon \rightarrow 0
$$

and hence $\Delta_{p}^{n} \phi(x) \leq 0$.

We proceed similarly, if $u-\phi$ has a strict maximum at $x$.

\section{The (AMVP) FOR THE PARABOLIC CASE}

The situation in the parabolic case is similar to that presented in the previous paragraph: we just have to use the proper cost function. As already observed, the choice disclosed in (1.11) is a good candidate since it yields for $p=2$ the classical mean value property for solutions of the heat equation. Thus, we shall denote:

$$
\pi_{p}(\varepsilon, u)(x, t)=\text { the unique } \pi \in \mathbb{R} \text { satisfying (1.11). }
$$

It is clear that the characterization, continuity and monotonicity of Theorems 2.1. 2.4 and 2.5 apply to $\pi_{p}(\varepsilon, u)(x, t)$, if we set $X=\overline{E_{\varepsilon}(x, t)}$ and $d \nu(y, s)=$ $|x-y|^{2} /(t-s)^{2} d y d s$. In particular, the heat mean value of $u$ is

$$
f_{E_{\varepsilon}(x, t)} u(y, s) d \nu(y, s)=\frac{1}{4 \varepsilon^{N}} \int_{E_{\varepsilon}(x, t)} u(y, s) d \nu(y, s)
$$


and the heat median of $u, \underset{E_{\varepsilon}(x, t)}{\mathrm{h}-\mathrm{med}} u$, is the unique root of the equation:

$$
\int_{E_{\varepsilon}^{\lambda,+}(x, t)} \frac{|x-y|^{2}}{(t-s)^{2}} d y d s=\int_{E_{\varepsilon}^{\lambda,-}(x, t)} \frac{|x-y|^{2}}{(t-s)^{2}} d y d s
$$

where

$$
E_{\varepsilon}^{\lambda, \pm}(x, t)=\left\{(y, s) \in E_{\varepsilon}(x, t): \lambda \lessgtr u(y, s)\right\} .
$$

The companion of Corollary 2.3 is the following result, that does not need an ad hoc proof.

Corollary 4.1. Let $1 \leq p<\infty, u \in C\left(\overline{E_{\varepsilon}(x, t)}\right)$ and define

$$
u_{\varepsilon}(z, \sigma)=u\left(x+\varepsilon z, t-\varepsilon^{2} \sigma\right),(z, \sigma) \in E,
$$

where

$$
E=\left\{(z, \sigma) \in \mathbb{R}^{N+1}: 0<\sigma<\frac{1}{4 \pi}, \Phi(z, \sigma)>1\right\}
$$

Then

$$
\pi_{p}(\varepsilon, u)(x, t)=\pi_{p}\left(1, u_{\varepsilon}\right)(0,0),
$$

where $\lambda=\pi_{p}\left(1, u_{\varepsilon}\right)(0,0)$ is the unique root of the equation

$$
\int_{E}\left|u_{\varepsilon}(z, \sigma)-\lambda\right|^{p-2}\left[u_{\varepsilon}(z, \sigma)-\lambda\right] d \nu(z, \sigma)=0 .
$$

Lemma 4.2. Let $1 \leq p \leq \infty$, pick $a \in \mathbb{R}$ and $\xi \in \mathbb{R}^{N} \backslash\{0\}$, and let $A$ be $a$ symmetric $N \times N$ matrix.

Consider the quadratic function $q: E_{\varepsilon}(x, t) \rightarrow \mathbb{R}$ defined by

$$
q(y, s)=q(x, t)+\xi \cdot(y-x)+a(s-t)+\frac{1}{2}\langle A(y-x), y-x\rangle
$$

for $(y, s) \in E_{\varepsilon}(x, t)$. Let $\pi_{p}(\varepsilon, q)$ be the heat $p$-mean of $q$ on $E_{\varepsilon}(x, t)$.

Then it holds that

$$
\begin{aligned}
& \pi_{p}(\varepsilon, q)=q(x, t)+ \\
& \frac{1}{4 \pi}\left(1-\frac{2}{N+p}\right)^{1+\frac{N+p}{2}}\left\{-a+\frac{N}{N+p-2}\left[\operatorname{tr}(A)+(p-2) \frac{\langle A \xi, \xi\rangle}{|\xi|^{2}}\right]\right\} \varepsilon^{2}+o\left(\varepsilon^{2}\right)
\end{aligned}
$$

as $\varepsilon \rightarrow 0$.

Proof. We proceed similarly to the proof of Lemma 3.1] Set

$$
\begin{aligned}
& q_{\varepsilon}(z, \sigma)=q\left(x+\varepsilon z, t-\varepsilon^{2} \sigma\right), \\
& v_{\varepsilon}(z, \sigma)=\frac{q\left(x+\varepsilon z, t-\varepsilon^{2} \sigma\right)-q(x, t)}{\varepsilon} \text { and } v(z, \sigma)=\xi \cdot z .
\end{aligned}
$$

We know that

$$
\pi_{p}(\varepsilon, q)(x, t)=\pi_{p}\left(1, q_{\varepsilon}\right)(0,0)
$$

thus, Proposition 2.7 implies that

$$
\frac{\pi_{p}(\varepsilon, q)(x, t)-q(x, t)}{\varepsilon}=\pi_{p}\left(1, v_{\varepsilon}\right)(0,0),
$$

and $v_{\varepsilon}$ converges to $v$ uniformly on $E$ as $\varepsilon \rightarrow 0$. Theorem 2.4 then yields that

$$
\lim _{\varepsilon \rightarrow 0} \frac{\pi_{p}(\varepsilon, q)(x, t)-q(x, t)}{\varepsilon}=\pi_{p}(1, v)(0,0),
$$

and $\pi_{p}(1, v)=0$, since it is the unique solution $\lambda$ of

$$
\int_{E}|v(z, \sigma)-\lambda|^{p-2}[v(z, \sigma)-\lambda] d \nu(z, \sigma)=0,
$$


for $1 \leq p<\infty$, and for $p=\infty$ maximizes the quantity

$$
\max (|\xi|-\lambda, \lambda+|\xi|)
$$

for $\lambda \in \mathbb{R}$.

As before, set

$$
\delta_{\varepsilon}=\frac{\pi_{p}(\varepsilon, q)(x, t)-q(x, t)}{\varepsilon^{2}} .
$$

Case $\mathbf{1}<\mathbf{p}<\infty$. By some manipulations, we get that

$$
\int_{E} h\left(\xi \cdot z+\varepsilon\left[-a \sigma+\langle A z, z\rangle / 2-\delta_{\varepsilon}\right]\right) d \nu(z, \sigma)=0,
$$

where $h$ is the function already defined. Without loss of generality, we assume that $|\xi|=1$, apply the change of variables $z=R y$, where $R$ is a rotation matrix such that ${ }^{t} R \xi=e_{1}$, and set $C={ }^{t} R A R$ to obtain that

$$
\int_{E} \frac{h\left(y_{1}+\varepsilon\left[-a \sigma+\langle C y, y\rangle / 2-\delta_{\varepsilon}\right]\right)-h\left(y_{1}\right)}{\varepsilon} d \nu(y, \sigma),
$$

since $\int_{E} h\left(y_{1}\right)|y|^{2} / \sigma^{2} d y d \sigma=0$. Thus, by proceeding as before, we have that

$$
\begin{aligned}
& \delta_{\varepsilon} \int_{E}\left\{\int_{0}^{1} h^{\prime}\left(y_{1}+\tau \varepsilon\left[-a \sigma+\langle C y, y\rangle / 2-\delta_{\varepsilon}\right]\right) d \tau\right\} d \nu(y, \sigma)= \\
& \int_{E}\left\{\int_{0}^{1} h^{\prime}\left(y_{1}+\tau \varepsilon\left[-a \sigma+\langle C y, y\rangle / 2-\delta_{\varepsilon}\right]\right) d \tau\right\}[-a \sigma+\langle C y, y\rangle / 2] d \nu(y, \sigma),
\end{aligned}
$$

and this implies that $\delta_{\varepsilon}$ is bounded by some constant (this is equal to $c+|a| / 4 \pi$ ).

If $2 \leq p<\infty$, it is easy to prove that, by the dominated convergence theorem, (any converging subsequence of) $\delta_{\varepsilon}$ converges to the number $\delta_{0}$ defined by

$$
\int_{E} h^{\prime}\left(y_{1}\right)\left[-a \sigma+\langle C y, y\rangle / 2-\delta_{0}\right] d \nu(y, \sigma)=0 .
$$

If $1<p<2$, we observe that

$$
\begin{aligned}
\left|\int_{0}^{1} h^{\prime}\left(y_{1}+\tau \varepsilon\left[-a \sigma+\langle C y, y\rangle / 2-\delta_{\varepsilon}\right]\right)\left[-a \sigma+\langle C y, y\rangle / 2-\delta_{\varepsilon}\right] d \tau\right| \leq \\
2(c+|a| / 4 \pi)|| y_{1}|-2(c+|a| / 4 \pi) \varepsilon|^{p-2}
\end{aligned}
$$

and

$$
\lim _{\varepsilon \rightarrow 0} \int_{E}|| y_{1}|-2(c+|a| / 4 \pi) \varepsilon|^{p-2} d \nu(y, \sigma)=\int_{B}\left|y_{1}\right|^{p-2} d \nu(y, \sigma) .
$$

If (any converging subsequence of) $\delta_{\varepsilon}$ converges to a number $\delta_{0}$, then the integrand in (3.2) converges pointwise to $h^{\prime}\left(y_{1}\right)\left[-a \sigma+\langle C y, y\rangle / 2-\delta_{0}\right]$, and hence we can conclude that (4.7) holds, by the generalized dominated convergence theorem (Theorem 5.4).

Therefore, by Lemma 5.3 we have that

$$
\begin{aligned}
& \lim _{\varepsilon \rightarrow 0} \delta_{\varepsilon}=\frac{\int_{E}\left|y_{1}\right|^{p-2}[-a \sigma+\langle C y, y\rangle / 2] d \nu(y, \sigma)}{\int_{E}\left|y_{1}\right|^{p-2} d \nu(y, \sigma)}= \\
& \frac{1}{4 \pi}\left(1-\frac{2}{N+p}\right)^{\frac{N+p}{2}+1}\left\{-a+\frac{N}{N+p-2}\left[\operatorname{tr}(C)+(p-2)\left\langle C e_{1}, e_{1}\right\rangle\right]\right\}= \\
& \quad \frac{1}{4 \pi}\left(1-\frac{2}{N+p}\right)^{\frac{N+p}{2}+1}\left\{-a+\frac{N}{N+p-2}\left[\operatorname{tr}(A)+(p-2) \frac{\langle A \xi, \xi\rangle}{|\xi|^{2}}\right]\right\}
\end{aligned}
$$

since $\left\langle C e_{1}, e_{1}\right\rangle=\left\langle A R e_{1}, R e_{1}\right\rangle$, with $R e_{1}=\xi /|\xi|$. 
Case $\mathbf{p}=\mathbf{1}$. By proceeding as in the proof of Lemma 3.1, it is easy to show that

where

$$
\int_{E_{\varepsilon}^{+}} \frac{|y|^{2}}{\sigma^{2}} d y d \sigma=\int_{E_{\varepsilon}^{-}} \frac{|y|^{2}}{\sigma^{2}} d y d \sigma
$$

$$
E_{\varepsilon}^{\mp}=\left\{(y, \sigma) \in E:-y_{1} \lessgtr 0,|\xi| y_{1}+\varepsilon[-a \sigma+\langle C y, y\rangle / 2] \lessgtr \varepsilon \delta_{\varepsilon}\right\},
$$

$R$ is the usual rotation matrix, and $C={ }^{t} R A R$.

Now, we assume that $|\xi|=1$ without loss of generality and use the change of variables

$$
y=\varepsilon z_{1} e_{1}+z^{\prime} \text { where } z^{\prime}=\left(0, z_{2}, \ldots, z_{N}\right)
$$

and take the limit as $\varepsilon \rightarrow 0$; similarly to the proof of Lemma 3.1 we obtain that

$$
\int_{E_{0}^{+}} \frac{|z|^{2}}{\sigma^{2}} d z d \sigma=\int_{E_{0}^{-}} \frac{|z|^{2}}{\sigma^{2}} d z d \sigma
$$

where $\delta_{0}$ is, as usual, the limit of $\delta_{\varepsilon}$ as $\delta \rightarrow 0$ and

$$
\begin{aligned}
E_{0}^{ \pm}=\left\{(z, \sigma) \in \mathbb{R}^{N+1}: 0 \leq\left|z^{\prime}\right|<\sqrt{-2 N \sigma \log (4 \pi \sigma)}, 0<\sigma<\frac{1}{4 \pi},\right. \\
\left.0 \lessgtr z_{1}, \delta_{0} \lessgtr z_{1}-a \sigma+\frac{\left\langle C z^{\prime}, z^{\prime}\right\rangle}{2}\right\} .
\end{aligned}
$$

Thus, $\delta_{0}$ results to be the solution of

$$
\int_{E^{\prime}}\left[\delta_{0}+a \sigma-\frac{1}{2}\left\langle C z^{\prime}, z^{\prime}\right\rangle\right] \frac{\left|z^{\prime}\right|^{2}}{\sigma^{2}} d z^{\prime} d \sigma=0,
$$

where

$$
E^{\prime}=\left\{\left(z^{\prime}, \sigma\right) \in \mathbb{R}^{N}: 0 \leq\left|z^{\prime}\right|<\sqrt{-2 N \sigma \log (4 \pi \sigma)}, 0<\sigma<\frac{1}{4 \pi}\right\}
$$

and hence

$$
\begin{aligned}
\delta_{0} \int_{E^{*}} r^{N} \sigma^{-2} d r d \sigma=-a \int_{E^{*}} & r^{N} \sigma^{-1} d r d \sigma+ \\
& \frac{1}{2(N-1)}\left[\operatorname{tr}(A)-\frac{\langle A \xi, \xi\rangle}{|\xi|^{2}}\right] \int_{E^{*}} r^{N+2} \sigma^{-2} d r d \sigma .
\end{aligned}
$$

Finally, Lemma 5.2 gives that

$$
\delta_{0}=\frac{1}{4 \pi}\left(\frac{N-1}{N+1}\right)^{\frac{N+1}{2}+1}\left\{-a+\frac{N}{N-1}\left[\operatorname{tr}(A)-\frac{\langle A \xi, \xi\rangle}{|\xi|^{2}}\right]\right\} .
$$

Case $\mathbf{p}=\infty$. For what we already showed at the beginning of this proof, we know that

$$
\begin{aligned}
\frac{\pi_{\infty}(\varepsilon, q)-q(x, t)}{\varepsilon}=\frac{1}{2} \min _{(z, \sigma) \in E}[\xi \cdot z+\varepsilon & (-a \sigma+\langle A z, z\rangle / 2)]+ \\
& \frac{1}{2} \max _{(z, \sigma) \in E}[\xi \cdot z+\varepsilon(-a \sigma+\langle A z, z\rangle / 2)] .
\end{aligned}
$$

Now, notice that if $\varepsilon$ is sufficiently small, since $\xi \neq 0$, the minimum and the maximum are attained at some points $\left(z_{\varepsilon}^{+}, \sigma_{\varepsilon}^{+}\right)$and $\left(z_{\varepsilon}^{-}, \sigma_{\varepsilon}^{-}\right)$on $\partial E$. Thus, there exist two Lagrange multipliers $\lambda_{\varepsilon}^{+}$and $\lambda_{\varepsilon}^{-}$such that the following three equations hold:

$$
\begin{gathered}
\xi+\varepsilon A z_{\varepsilon}^{ \pm}=\lambda_{\varepsilon}^{ \pm} z_{\varepsilon}^{ \pm}, \quad-\varepsilon a=\lambda_{\varepsilon}^{ \pm} N\left\{\log \left(4 \pi \sigma_{\varepsilon}^{ \pm}\right)+1\right\}, \\
\left|z_{\varepsilon}^{ \pm}\right|^{2}+2 N \sigma_{\varepsilon}^{ \pm} \log \left(4 \pi \sigma_{\varepsilon}^{ \pm}\right)=0 .
\end{gathered}
$$


Since

$$
\max _{(z, \sigma) \in E}(\xi \cdot z)=|\xi| \sqrt{\frac{N}{2 \pi e}} \text { and } \min _{(z, \sigma) \in E}(\xi \cdot z)=-|\xi| \sqrt{\frac{N}{2 \pi e}},
$$

a straightforward asymptotic analysis on the system (4.8) informs us that

$$
z_{\varepsilon}^{ \pm}= \pm \sqrt{\frac{N}{2 \pi e}} \frac{\xi}{|\xi|}+o(\varepsilon) \text { and } \sigma_{\varepsilon}^{ \pm}=\frac{1}{4 \pi e}+o(\varepsilon) \text { as } \varepsilon \rightarrow 0 .
$$

Therefore, we obtain:

$$
\begin{aligned}
& \lim _{\varepsilon \rightarrow 0} \frac{\pi_{\infty}(\varepsilon, q)-q(x, t)}{\varepsilon^{2}}= \\
& \lim _{\varepsilon \rightarrow 0}\left\{\xi \cdot \frac{z_{\varepsilon}^{-}+z_{\varepsilon}^{+}}{2 \varepsilon}-a \frac{\sigma_{\varepsilon}^{-}+\sigma_{\varepsilon}^{+}}{2}+\frac{\left\langle A z_{\varepsilon}^{-}, z_{\varepsilon}^{-}\right\rangle+\left\langle A z_{\varepsilon}^{+}, z_{\varepsilon}^{+}\right\rangle}{4}\right\}= \\
& \frac{1}{4 \pi e}\left(-a+N \frac{\langle A \xi, \xi\rangle}{|\xi|^{2}}\right),
\end{aligned}
$$

as desired.

Theorem 4.3 (Asymptotics for $\pi_{p}(\varepsilon, u)$ as $\varepsilon \rightarrow 0$ ). Let $1 \leq p \leq \infty$. Assume $(x, t) \in \Omega_{T}, u \in C^{2}\left(\Omega_{T}\right)$ and $\nabla u(x, t) \neq 0$.

Then

$$
\begin{aligned}
& \pi_{p}(\varepsilon, u)(x, t)=u(x, t)+ \\
& \quad \frac{1}{4 \pi}\left(1-\frac{2}{N+p}\right)^{\frac{N+p}{2}+1}\left\{-u_{t}(x, t)+\frac{N}{N+p-2} \Delta_{p}^{n} u(x, t)\right\} \varepsilon^{2}+o\left(\varepsilon^{2}\right),
\end{aligned}
$$

as $\varepsilon \rightarrow 0$.

Proof. Let $\varepsilon>0$ be such that $\overline{E_{\varepsilon}(x, t)} \subset \Omega_{T}$ and consider the function $q(y, s)$ in Lemma 4.2 with $q(x, t)=u(x, t), a=u_{t}(x, t), \xi=\nabla u(x, t)$, and $A=\nabla^{2} u(x, t)$; then, set $u_{\varepsilon}(z, \sigma)=u\left(x+\varepsilon z, t-\varepsilon^{2} \sigma\right)$ and $q_{\varepsilon}(z, \sigma)=u\left(x+\varepsilon z, t-\varepsilon^{2} \sigma\right)$.

Since $u \in C^{2}\left(\Omega_{T}\right)$, for every $\eta>0$ there exists $\varepsilon_{\eta}>0$ such that

$$
\left|u_{\varepsilon}(z, \sigma)-q_{\varepsilon}(z, \sigma)\right|<\eta \varepsilon^{2} \text { for every } z \in \bar{E} \text { and } 0<\varepsilon<\varepsilon_{\eta} .
$$

Thus, by Proposition 2.7. (4.4) and Theorem 2.5.

$$
\begin{aligned}
\frac{\pi_{p}(\varepsilon, q)(x, t)-q(x, t)}{\varepsilon^{2}}-\eta \leq \frac{\pi_{p}(\varepsilon, u)(x, t)-q(x, t)}{\varepsilon^{2}} & \leq \\
& \frac{\pi_{p}(\varepsilon, q)(x, t)-q(x, t)}{\varepsilon^{2}}+\eta .
\end{aligned}
$$

Therefore, Lemma 4.2 implies that

$$
\begin{gathered}
\frac{1}{4 \pi}\left(1-\frac{2}{N+p}\right)^{\frac{N+p}{2}+1}\left\{-u_{t}(x, t)+\frac{N}{N+p-2} \Delta_{p}^{n} u(x, t)\right\}-\eta \leq \\
\liminf _{\varepsilon \rightarrow 0} \frac{\pi_{p}(\varepsilon, u)(x, t)-q(x, t)}{\varepsilon^{2}} \leq \limsup _{\varepsilon \rightarrow 0} \frac{\pi_{p}(\varepsilon, u)(x, t)-q(x, t)}{\varepsilon^{2}} \leq \\
\frac{1}{4 \pi}\left(1-\frac{2}{N+p}\right)^{\frac{N+p}{2}+1}\left\{-u_{t}(x, t)+\frac{N}{N+p-2} \Delta_{p}^{n} u(x, t)\right\}+\eta .
\end{gathered}
$$

The desired conclusion follows at once, since $\eta$ is arbitrary.

Corollary 4.4. Let $u \in C^{2}\left(\Omega_{T}\right)$. The following assertions are equivalent:

(i) $-u_{t}(x, t)+\frac{N}{N+p-2} \Delta_{p}^{n} u(x, t)=0$,

(ii) $u(x)=\pi_{p}(\varepsilon, u)(x, t)+o\left(\varepsilon^{2}\right)$ as $\varepsilon \rightarrow 0$, 
at any point $(x, t) \in \Omega_{T}$ such that $\nabla u(x, t) \neq 0$.

We do not provide the proof of Theorem 1.2 since is a straightforward readaptation of that of Theorem 1.1, once the following definitions are established.

A function $u \in C\left(\Omega_{T}\right)$ is a viscosity solution of $u_{t}=\frac{N}{N+p-2} \Delta_{p}^{n} u$ in $\Omega_{T}$, if both of the following requisites hold at every $(x, t) \in \Omega_{T}$ :

(i) for any function $\phi$ of class $C^{2}$ near $(x, t)$ such that $u-\phi$ has a strict minimum at $(x, t)$ with $u(x, t)=\phi(x, t)$ and $\nabla \phi(x, t) \neq 0$, there holds that $\frac{N}{N+p-2} \Delta_{p}^{n} u(x, t) \leq u_{t}(x, t)$

(ii) for any function $\phi$ of class $C^{2}$ near $(x, t)$ such that $u-\phi$ has a strict maximum at $(x, t)$ with $u(x, t)=\phi(x, t)$ and $\nabla \phi(x, t) \neq 0$, there holds that $\frac{N}{N+p-2} \Delta_{p}^{n} u(x, t) \geq u_{t}(x, t)$.

We say that a function $u \in C\left(\Omega_{T}\right)$ satisfies at $(x, t) \in \Omega_{T}$ the asymptotic mean value property (AMVP)

$$
u(x, t)=\pi_{p}(\varepsilon, u)(x, t)+o\left(\varepsilon^{2}\right) \text { as } \varepsilon \rightarrow 0
$$

in the viscosity sense if both of the following requisites hold:

(a) for any function $\phi$ of class $C^{2}$ near $(x, t)$ such that $u-\phi$ has a strict minimum at $(x, t)$ with $u(x, t)=\phi(x, t)$ and $\nabla \phi(x, t) \neq 0$, there holds that

$$
\phi(x, t) \geq \pi_{p}(\varepsilon, \phi)(x, t)+o\left(\varepsilon^{2}\right) \text { as } \varepsilon \rightarrow 0 ;
$$

(b) for any function $\phi$ of class $C^{2}$ near $(x, t)$ such that $u-\phi$ has a strict maximum at $(x, t)$ with $u(x, t)=\phi(x, t)$ and $\nabla \phi(x, t) \neq 0$, there holds that

$$
\phi(x, t) \leq \pi_{p}(\varepsilon, \phi)(x, t)+o\left(\varepsilon^{2}\right) \text { as } \varepsilon \rightarrow 0 .
$$

\section{USEFUL INTEGRALS}

We begin with the computation of some useful integrals.

Lemma 5.1. Let $\mathbb{S}^{N-1}$ be the unit sphere in $\mathbb{R}^{N}$. Let $\xi \in \mathbb{R}^{N} \backslash\{0\}$ and $A$ be an $N \times N$ symmetric matrix. Then for $1<p<\infty$ we have that

$$
\frac{\int_{\mathbb{S}^{N-1}}|\xi \cdot y|^{p-2}\langle A y, y\rangle d S_{y}}{\int_{\mathbb{S}^{N-1}}|\xi \cdot y|^{p-2} d S_{y}}=\frac{1}{N+p-2}\left\{\operatorname{tr}(A)+(p-2) \frac{\langle A \xi, \xi\rangle}{|\xi|^{2}}\right\}
$$

and

$$
\frac{\int_{B}|\xi \cdot y|^{p-2}\langle A y, y\rangle d y}{\int_{B}|\xi \cdot y|^{p-2} d y}=\frac{1}{N+p}\left\{\operatorname{tr}(A)+(p-2) \frac{\langle A \xi, \xi\rangle}{|\xi|^{2}}\right\} .
$$

Proof. Let $R$ be a rotation matrix such that ${ }^{t} R \xi=|\xi| e_{1}$; by the change of variables $y=R \theta$, we have that

$$
\frac{\int_{\mathbb{S}^{N-1}}|\xi \cdot y|^{p-2}\langle A y, y\rangle d S_{y}}{\int_{\mathbb{S}^{N-1}}|\xi \cdot y|^{p-2} d S_{y}}=\frac{\int_{\mathbb{S}^{N-1}}\left|\theta_{1}\right|^{p-2}\left\langle\left({ }^{t} R A R\right) \theta, \theta\right\rangle d S_{\theta}}{\int_{\mathbb{S}^{N-1}}\left|\theta_{1}\right|^{p-2} d S_{\theta}} .
$$

On the other hand,

$$
\begin{aligned}
& \int_{\mathbb{S}^{N-1}}\left|\theta_{1}\right|^{p-2} \theta_{i} \theta_{j} d S_{\theta}=\int_{B} \frac{\partial}{\partial y_{j}}\left(\left|y_{1}\right|^{p-2} y_{i}\right) d y= \\
& {\left[\delta_{i j}+(p-2) \delta_{i 1} \delta_{1 j}\right] \int_{B}\left|y_{1}\right|^{p-2} d y=\frac{\delta_{i j}+(p-2) \delta_{i 1} \delta_{1 j}}{N+p-2} \int_{\mathbb{S}^{N-1}}\left|\theta_{1}\right|^{p-2} d S_{\theta}}
\end{aligned}
$$


where we have used the divergence theorem in the first equality. Therefore, we obtain that

$$
\begin{gathered}
\frac{\int_{\mathbb{S}^{N-1}}|\xi \cdot y|^{p-2}\langle A y, y\rangle d S_{y}}{\int_{\mathbb{S}^{N-1}}|\xi \cdot y|^{p-2} d S_{y}}= \\
\frac{\operatorname{tr}\left({ }^{t} R A R\right)+(p-2)\left\langle\left({ }^{t} R A R\right) e_{1}, e_{1}\right\rangle}{N+p-2}= \\
\frac{1}{N+p-2}\left\{\operatorname{tr}(A)+(p-2) \frac{\langle A \xi, \xi\rangle}{|\xi|^{2}}\right\} .
\end{gathered}
$$

Formula (5.2) easily follows from (5.1).

Lemma 5.2. Let $\alpha>0$ and $\beta<\alpha+1$ be real numbers and let

$$
E_{*}=\left\{(r, \sigma) \in \mathbb{R}^{2}: 0<r<\sqrt{-2 N \sigma \log (4 \pi \sigma)}, 0<\sigma<\frac{1}{4 \pi}\right\} .
$$

Then

$$
\int_{E_{*}} r^{2 \alpha-1} \sigma^{-\beta} d r d \sigma=\frac{2^{2 \beta-\alpha-3} \pi^{\beta-\alpha-1} N^{\alpha}}{\alpha(\alpha-\beta+1)^{\alpha+1}} \Gamma(\alpha+1) .
$$

Proof. The result follows from the calculations:

$$
\begin{gathered}
\int_{E_{*}} r^{2 \alpha-1} \sigma^{-\beta} d r d \sigma=\frac{1}{2 \alpha} \int_{0}^{\frac{1}{4 \pi}} \sigma^{-\beta}\{-2 N \sigma \log (4 \pi \sigma)\}^{\alpha} d \sigma= \\
\frac{2^{2 \beta-\alpha-3} \pi^{\beta-\alpha-1} N^{\alpha}}{\alpha} \int_{0}^{\infty} \tau^{\alpha} e^{-(\alpha-\beta+1) \tau} d \tau= \\
\frac{2^{2 \beta-\alpha-3} \pi^{\beta-\alpha-1} N^{\alpha}}{\alpha(\alpha-\beta+1)^{\alpha+1}} \int_{0}^{\infty} \tau^{\alpha} e^{-\tau} d \tau ;
\end{gathered}
$$

in the second equality we used the substitution $4 \pi \sigma=e^{-\tau}$.

Lemma 5.3. Let $\xi$ and $A$ be as in Lemma 5.1. Then for $1<p<\infty$ we have that

$$
\frac{\int_{E}|\xi \cdot z|^{p-2} \sigma d \nu(z, \sigma)}{\int_{E}|\xi \cdot z|^{p-2} d \nu(z, \sigma)}=\frac{1}{4 \pi}\left(\frac{N+p-2}{N+p}\right)^{1+\frac{N+p}{2}}
$$

and

$$
\begin{aligned}
& \frac{\int_{E}|\xi \cdot z|^{p-2}\langle A z, z\rangle d \nu(z, \sigma)}{\int_{E}|\xi \cdot z|^{p-2} d \nu(z, \sigma)}= \\
& \frac{1}{2 \pi} \frac{N}{N+p-2}\left(\frac{N+p-2}{N+p}\right)^{1+\frac{N+p}{2}}\left\{\operatorname{tr}(A)+(p-2) \frac{\langle A \xi, \xi\rangle}{|\xi|^{2}}\right\} .
\end{aligned}
$$

Proof. By using spherical coordinates, we calculate that

and

$$
\frac{\int_{E}|\xi \cdot z|^{p-2} \sigma d \nu(z, \sigma)}{\int_{E}|\xi \cdot z|^{p-2} d \nu(z, \sigma)}=\frac{\int_{E_{*}} r^{p+N-1} \sigma^{-1} d r d \sigma}{\int_{E_{*}} r^{p+N-1} \sigma^{-2} d r d \sigma}
$$

$$
\frac{\int_{E}|\xi \cdot z|^{p-2}\langle A z, z\rangle d \nu(z, \sigma)}{\int_{E}|\xi \cdot z|^{p-2} d \nu(z, \sigma)}=\frac{\int_{E_{*}} r^{p+N+1} \sigma^{-2} d r d \sigma}{\int_{E_{*}} r^{p+N-1} \sigma^{-2} d r d \sigma} \frac{\int_{\mathbb{S}^{N-1}}|\xi \cdot y|^{p-2}\langle A y, y\rangle d S_{y}}{\int_{\mathbb{S}^{N-1}}|\xi \cdot y|^{p-2} d S_{y}} .
$$

Thus, (5.4) and (5.5) follow from the calculations

$$
\frac{\int_{E_{*}} r^{p+N-1} \sigma^{-1} d r d \sigma}{\int_{E_{*}} r^{p+N-1} \sigma^{-2} d r d \sigma}
$$

and (5.1). 
For the reader's convenience, we recall the generalized dominated convergence theorem (see [10] for instance), that is needed for the proofs of Lemmas 3.1] and 4.2 .

Theorem 5.4 (Generalized dominated convergence theorem). Let $(X, \nu)$ be a measure space and let $\left\{f_{n}\right\}_{n \in \mathbb{N}}$ and $\left\{g_{n}\right\}_{n \in \mathbb{N}}$ be sequences of measurable functions on $X$ such that

(i) $f_{n}$ converges to a measurable function $f$ a.e. on $X$ as $n \rightarrow \infty$;

(ii) each $g_{n} \in L^{1}(X, \nu)$ and $g_{n}$ converges to a function $g$ in $L^{1}(X, \nu)$ a.e. on $X$ as $n \rightarrow \infty$;

(iii) $\left|f_{n}\right| \leq g_{n}$ a.e. on $X$ for all $n \in \mathbb{N}$;

(iv) $\lim _{n \rightarrow \infty} \int_{X} g_{n} d \nu=\int_{X} g d \nu$.

Then, we have that

$$
\lim _{n \rightarrow \infty} \int_{X} f_{n} d \nu=\int_{X} f d \nu
$$

Acknowledgments. The second author was supported by a PRIN grant of the italian MIUR and the Gruppo Nazionale per l'Analisi Matematica, la Probabilità e le loro Applicazioni (GNAMPA) of the Italian Istituto Nazionale di Alta Matematica (INdAM).

\section{REFERENCES}

[1] A. Arroyo and J. G. Llorente, On the asymptotic mean value property for planar p-harmonic functions, arXiv:1507.02108v1, July 2015.

[2] A. Bonfiglioli and E. Lanconelli, Subharmonic functions in sub-Riemannian Settings, J. Eur. Math. Soc., 15 (2013), 387-441.

[3] A. Bonfiglioli, E. Lanconelli and F. Uguzzoni, Stratified Lie groups and potential theory for their sub-Laplacians, Springer, 2007.

[4] L. C. Evans, Partial Differential Equations, Graduate Studies in Mathematics 19, Amer. Math. Soc., Providence, IR, 1998.

[5] M. Falcone, S. Finzi Vita, T. Giorgi and R. G. Smits, A semi-lagrangian scheme for the game p-laplacian via p-averaging, Appl. Numer. Math. 73 (2013), 63-80.

[6] T. Giorgi and R. G. Smits, Mean value property for p-harmonic functions, Proc. Amer. Math. Soc., 140 (2012), 2453-2463.

[7] D. Hartenstine and M. Rudd, Asymptotic statistical characterizations of p-harmonic functions of two variables, Rocky Mountain J. Math. 41 (2011), 493-504.

[8] P. Juutinen, P. Lindqvist and J. J. Manfredi, On the equivalence of viscosity solutions and weak solutions for a quasi-linear elliptic equation, SIAM, J. Math. Anal. 33 (2001), 699-717.

[9] B. Kawohl, J. J. Manfredi and M. Parviainen, Solutions of nonlinear PDEs in the sense of averages, J. Math. Pures Appl., 97 (2012), 173-188.

[10] E. H. Lieb and M. Loss, Analysis: Second Edition, Amer. Math. Soc., 2001.

[11] P. Lindqvist and J. J. Manfredi, On the mean value property for the $p$-Laplace equation in the plane, Proc. Amer. Math. Soc. 144 (2016), no. 1, 143-149.

[12] H. Luiro, M. Parviainen and E. Saksman, On the existence and uniqueness of p-harmonious functions, Differ. Integral Equ., 27 (2014), 201-216.

[13] J. J. Manfredi, M. Parviainen and J. D. Rossi, An asymptotic mean value characterization for a class of nonlinear parabolic equations related to tug-of-war games, SIAM J. Math. Anal., 42 (2010), 2058-2081.

[14] J. J. Manfredi, M. Parviainen and J. D. Rossi, An asymptotic mean value characterization for p-harmonic functions, Proc. Amer. Math. Soc., 138 (2010), 881-889.

[15] J. J. Manfredi, M. Parviainen and J. D. Rossi, On the definition and properties of pharmonious functions, Ann. Sc. Norm. Super. Pisa Cl. Sci. (5) 11 (2012), no. 2, 215-241.

[16] S. G. Noah, The median of a continuous function, Real Analysis Exchange, 33 (2008), 269274 .

[17] M. Rudd and H. Van Dyke, Median values, 1-harmonic functions, and functions of least gradient, Commun. Pure Appl. Anal. 12 (2013), 711-719.

[18] J. Shao, Mathematical Statistics, Springer-Verlag, New York, 1999.

[19] D. W. Stroock, Probability Theory, an Analytic View, Cambridge UP, Cambridge, 1993. 
Department of Systems Innovation Graduate School of Engineering Science, Osaka University, 1-3 Machikaneyama, Toyonaka, Osaka 560-8531, Japan

E-mail address: ishiwata@sigmath.es.osaka-u.ac.jp

Dipartimento di Matematica "U. Dini", Università a di Firenze, viale Morgagni 67/A, 50134 FirenZE, ITALY

E-mail address: magnanin@math.unifi.it, paolo.salani@math.unifi.it

$U R L:$ http://web.math.unifi.it/users/magnanin

Faculty of Mechanical Engineering, Institute of Science and Engineering, Kanazawa University, KaKuma, KANAZAWA, IshiKAWA 920-1192, JAPAN

E-mail address: wadade@se.kanazawa-u.ac.jp 